\title{
Electrostatic screening and Friedel oscillations in semiconducting nanotubes
}

\author{
A.V. Chaplik, L.I. Magarill, and R.Z. Vitlina \\ Institute of Semiconductor Physics, Novosibirsk 630090, Russia \\ E-mail: magarill_lev@hotmail.com \\ Received April 8, 2008
}

\begin{abstract}
In $3 \mathrm{D}$ and $2 \mathrm{D}$ electronic systems the singular contribution to the static permittivity $\varepsilon$ (Kohn singularity) is a small correction to the regular part of $\varepsilon$ but it results in the leading term in asymptotic behavior of the screened potential (Friedel oscillations). In the present letter we show that for nanotubes quite different results are valid: $\varepsilon$ becomes infinitely large at the singular point and the Friedel oscillations do not play the dominant role in the screening at the large distances. Moreover, the zero and highest cylindrical harmonics of the effective potential are screened by quite different mechanisms.
\end{abstract}

PACS: 73.63.Fg Nanotubes.

Keywords: static permittivity, Friedel oscillations, nanotubes.

Collective effects in quasi-1D systems are remarkable by a number of distinguishing features. Probably the most non-trivial one relates to the strictly 1D system of interacting fermions with the linear single particle dispersion law (the Luttinger model). However, there exist also different $1 \mathrm{D}$ objects not described in terms of the Luttinger liquid first of all due to necessity to account for the transversal subbands and transitions between them. The corresponding example is a nanotube - a hollow cylinder with 2D electron gas on its surface. As usually, availability of the mobile electrons results in renormalization of the electron-electron interaction, i.e., screening. The specific form of the screened $e-e$ interaction is determined by the effective dimension and by the energy spectrum of the electron gas. As is known in 3D plasma the bare Coulomb interaction is replaced (due to screening) by the Yukawa law plus the Friedel oscillations $\cos \left(2 p_{F} r\right) / r^{3}$ if the electron gas is degenerate; here $p_{F}$ is the Fermi momentum, $r$ is the distance from a point charge, and $\hbar=1$. Similar results in the $2 \mathrm{D}$ case read: the regular part of the screened potential at $r \rightarrow \infty$ is $Q a_{B}^{2} / r^{3}$ [1], where $Q$ is the initial point charge, $a_{B}$ is the effective Bohr radius, whereas the oscillating contribution is proportional to $\cos \left(2 p_{F} r-\pi / 4\right) / r^{2}$. We see that both in 3D and in $2 \mathrm{D}$ problems the Kohn singularity of $\varepsilon(k)$ at $k=2 p_{F}$ gives the leading term of the screened potential for $r \rightarrow \infty$; here $\varepsilon(k)$ is the static permittivity depending on the momentum $k$.

Consider now a nanotube with the semiconductor type of the single particle energy spectrum:

$$
\varepsilon_{p, l}=\frac{p^{2}}{2 m}+B l^{2} ; B \equiv \frac{1}{2 m a^{2}}, l=0, \pm 1, \pm 2, \ldots
$$

Here $p$ is the electron momentum parallel to the tube axis, $l$ is the number of the subband, $m$ is the effective mass and $a$ is the nanotube radius. Thus, the problem in question differs from the similar one for a planar 2D system by only quantization of one of the component of momentum: $p_{x}=l / a$. The considered system is infinite and uniform in $z$-direction (the nanotube axis) and periodic and uniform in the azimuthal direction $\varphi$. Hence, the electron Green function $G$ depends on only differences $z-z^{\prime}, \varphi-\varphi^{\prime}$. In the Fourier representation $G$ is diagonal: $G\left(p, p^{\prime} ; l, l^{\prime}\right)=\delta_{p p^{\prime}} \delta_{l l^{\prime}} G(p, l)$.

In what follows we will apply the standard diagram technique to find the $e-e$ interaction renormalized by screening and we will use the linear theory of screening. We are aware that in strictly 1D system with linear single-particle dispersion law the Luttinger liquid model is valid. The linear screening theory relates to only longwave length limit. This theory breaks down for the momentum of the order of $p_{F}$ just when the Friedel oscillations become essential [2-4]*.

* There is a misprint in the Eq.(8) of Ref. 2. Asymptotic behavior of the screened potential $U_{\text {eff }}$ must be $1 /|x| \ln { }^{2}|x / d|$ rather than $1 /|x| \ln |x / d|$, otherwise $\int U_{\text {eff }}(x) d x$ diverges while the Fourier component $\bar{U}_{\text {eff }}(q)$ remains finite at $q \rightarrow 0$. 
However we actually deal with 2D object and we use the parabolic dispersion law for electrons. For such a situation the Luttinger model is not applicable and we do not have any other instrument except RPA to investigate the problem. After that it is not surprising that our results for the Friedel contribution to the screened potential differ qualitatively from the ones obtained in [2-4] and also [5].

The main difference is dependence of our effective (screened) potential not only on $z$ (coordinate along the tube axis) but also on $\varphi$ - azimuthal separation between two electrons on the surface of a hollow cylinder. We discovered that average potential (zero cylindrical harmonic) and all the other harmonics are screened qualitatively different.

We guess that in the present situation - there is an exactly solvable model but for only strictly 1D system with linear dispersion law and an approximate method for general case - it is worth to find general results within RPA and to see what experiments will show. This will be done in what follows.

The Gell-Mann-Brueckner [6] chain of electron loops determining the Fourier component of the screened $e-e$ interaction $V(k, n)$ comes to the geometrical progression:

$$
V(k, n)=\frac{V^{(0)}(k, n)}{1+V^{(0)}(k, n) \Pi(\omega ; k, n)},
$$

where $V^{(0)}$ is the bare Coulomb interaction:

$$
\begin{aligned}
& V^{(0)}(k, n)=\widetilde{e}^{2} \int_{-\infty}^{\infty} \int_{0}^{2 \pi} \frac{\mathrm{e}^{-i k z-i n \varphi} d z d \varphi}{\sqrt{z^{2}+4 a^{2} \sin ^{2}(\varphi / 2)}}= \\
& =4 \pi \tilde{e}^{2} I_{n}(|k| a) K_{n}(|k| a) .
\end{aligned}
$$

Here $\tilde{e}^{2}=e^{2} / \chi, \chi$ is the background dielectric constant, $I_{n}, K_{n}$ are the modified Bessel functions of the 1 th and the 3rd type. The polarization operator (electron loop) has a form

$$
\begin{array}{r}
\Pi(\omega ; k, n)=\frac{1}{2 \pi^{2}} \sum_{l=-\infty}^{\infty} \int_{-\infty}^{\infty} d p \frac{f_{p-k, l-n}-f_{p, l}}{\varepsilon_{p, l}-\varepsilon_{p-k, l-n}-\omega-i \delta} \\
(\delta=+0),
\end{array}
$$

where $f_{p, l} \equiv f\left(\varepsilon_{p, l}\right)$ are the Fermi occupation numbers.

We are interesting here in the static screening and we put from now on $\omega=0$. By transforming $V(k, n)$ from Eq. (2) back to $z$-space we obtain the expansion of the screened interaction in cylindrical harmonics. As $V^{(0)}(k, n)$ and $\Pi(0 ; k, n)$ are even functions of $n$ we come to the series

$$
\begin{aligned}
& V(z, \varphi)=\int_{-\infty}^{\infty} \frac{d k}{(2 \pi)^{2}} \exp (i k z) \times \\
& \times\left[V(k, 0)+2 \sum_{n=1}^{\infty} V(k, n) \cos (n \varphi)\right] .
\end{aligned}
$$

The slowest decreasing term at $|z| \rightarrow \infty$ is given by the zero harmonic and reads

$$
V_{0}(z)=\int_{0}^{\infty} \frac{d k}{\pi} \cos (k z) \frac{4 \pi \tilde{e}^{2} I_{0}(k a) K_{0}(k a)}{1+4 \pi \tilde{e}^{2} \Pi_{0}(k) I_{0}(k a) K_{0}(k a)},
$$

where

$$
\Pi_{0}(k) \equiv \Pi(0 ; k, 0)=\frac{m}{\pi^{2} k} \sum_{-L}^{L} \ln \left|\frac{k+2 p_{l}}{k-2 p_{l}}\right|,
$$

$L$ is the number of the highest occupied subband at zero temperature, $p_{l}=\sqrt{2 m\left(E_{F}-B l^{2}\right)}$ is the Fermi momentum of the lth subband. Eq. (7) demonstrates the qualitative difference of the quasi-1D problem and the 2D and 3D problems. In 2D and 3D systems the Kohn singularity at $k=2 p_{F}$ gives small corrections to the regular part of the polarization operator: $\xi \ln |\xi|$ in 3D [7] and $\sqrt{|\xi|}$ in 2D case, where $\xi=\left(k-2 p_{F}\right) / 2 p_{F} \ll 1$. But in 1D situation $\Pi_{0}(k)$ becomes infinitely large at $k=2 p_{l}$ and the renormalized interaction vanishes at these points.

To calculate $V_{0}(z)$ in the regime $|z| \gg>a$ we write in the Eq. (6) $\cos (k z)=\operatorname{Re}(\exp (i k z))$, and turn the path of integration to the upper imaginary semiaxis. At large $|z|$ we have two contributions to $V_{0}(z): \bar{V}_{0}$ from the point $k=0$, where $K_{0}$ has logarithmic singularity but $\Pi_{0}$ remains finite, and a number of the Kohn contributions from the points $k=2 p_{l}$ resulting in the Friedel oscillations $\widetilde{V}_{0}(z)$. For the nonoscillating part $\bar{V}_{0}(z)$ we get the expansion in inverse powers of the value $\Lambda \equiv \ln (2|z| / a)-C$ ( $C$ is the Euler constant)

$$
\bar{V}_{0}(z)=\frac{\tilde{e}^{2}}{z}\left(\frac{m a_{B}}{4 \pi \kappa_{0} \Lambda}\right)^{2}\left(1-\frac{m a_{B} / \pi \kappa_{0}+4 C}{2 \Lambda}+\ldots\right),
$$

where

$$
\kappa_{0} \equiv \Pi_{0}(k \rightarrow 0)=m\left[1 / p_{0}+2 \sum_{l=1}^{L}\left(1 / p_{l}\right)\right] / \pi^{2} ;
$$

$\pi \kappa_{0}$ is the sum of the partial density of states in occupied subbands. Thus, the Coulomb interaction in nanotubes is screened rather weakly, $\bar{V}_{0}(z) \sim e^{2} /\left(z \ln ^{2}(z / a)\right)$.

To find the oscillating part $\widetilde{V}_{0}(z)$ we consider a small vicinity of the point $k=2 p_{l}$ and note that the factor $\tilde{e}^{2} I_{0} K_{0}$ in the integrand of Eq. (6) cancels. Thus, we see yet another peculiarity of the 1D problem: Friedel oscillations of the screened potential do not depend on the charge! (see below Eqs. (9), (18)). This is due to infinitely large magnitude of $\Pi_{0}(k)$ at $k=2 p_{l}$. Then one can introduce the parameter $\xi=\left(k-2 p_{l}\right) / 2 p_{l}$ and take the integral over $\xi$ from $-\infty$ till $\infty$. By the same shift of the integration path as described above we arrive at the series in powers of $1 / \ln \left(4 p_{l} z\right)$ : 


$$
\begin{aligned}
& \widetilde{V}_{0}(z)= \\
& =-\sum_{l=-L}^{l=L} \frac{2 \pi^{2} p_{l}}{m} \frac{\cos \left(2 p_{l} z\right)}{z \ln ^{2}\left(4 p_{l} z\right)}\left[1-\frac{2 C}{\ln \left(4 p_{l} z\right)}+\ldots\right]
\end{aligned}
$$

We see that the amplitude of the Friedel oscillations in nanotubes decreases with increasing distance not slower than the regular part $\bar{V}_{0}(z)$ does. Moreover, the ratio $\widetilde{V}_{0} / \bar{V}_{0}$ at any $z$ in the order of magnitude can be estimated as $1 /\left(p_{F} a_{B}\right)$, and for «metallic» limit of the dense electron gas $\left(p_{F} a_{B} \gg 1\right)$ the Friedel oscillations become negligibly small.

Qualitatively different results are obtained for nonzero harmonics. The factor $I_{n}(k a) K_{n}(k a)$ for $n \neq 0$ tends to the constant $1 / 2 n$ for $k \rightarrow 0$ and $\Pi(k, n)$ at $k=0$ remains finite either. Thus, all non-zero harmonics of the Coulomb interaction undergo the screening of the dielectric type, that is, their dependence on the distance $z$ coincides with that of the bare Coulomb law. Indeed, from Eq. (3) we have for the $n$th harmonic of the bare interaction

$$
\begin{aligned}
& V_{n}^{(0)}(z)=\frac{\widetilde{e}^{2}}{\pi a} Q_{n-1 / 2}\left(1+\frac{z^{2}}{2 a^{2}}\right), \\
& V_{n}^{(0)}(z>>a) \simeq \frac{\Gamma(n+1 / 2)}{\sqrt{\pi} n !} \frac{\tilde{e}^{2}}{z}\left(\frac{a}{z}\right)^{2 n},
\end{aligned}
$$

where $Q_{V}$ is the spherical function of the second type. The regular part of the screened interaction stems again from the region of small $k$. Eq. (4) gives:

$\operatorname{Im}\left(\left.\Pi(k, n)\right|_{k=0}=0\right.$,

$\operatorname{Re}(\Pi(k, n))=\frac{m}{2 \pi^{2} k} \sum_{-L}^{L} \ln \left|\frac{\left(k^{2} a^{2}+n^{2}+2 k p_{l} a^{2}\right)^{2}-4 n^{2} l^{2}}{\left(k^{2} a^{2}+n^{2}-2 k p_{l} a^{2}\right)^{2}-4 n^{2} l^{2}}\right|$

and there are two possibilities for the quantity $\kappa_{n} \equiv \Pi(0, n)$. If the subband occupation for $T=0$ terminates at $l= \pm L$ and $n^{2}>4 L^{2}$, then

$$
\kappa_{n}=\frac{4 m a^{2}}{\pi^{2}} \sum_{l=-L}^{L} \frac{p_{l}}{n^{2}-4 l^{2}} .
$$

Otherwise, for $n^{2} \leq 4 L^{2}$ and $n$ even the $n$th harmonic of the potential couples the degenerate states $l_{0}=n / 2$ and $l_{0}=-n / 2$. In this case we calculate the limit of the uncertainty in Eq. (11) at $n=2 l_{0}, k \rightarrow 0$ and find

$$
\Pi_{n=2 l_{0}}=\frac{2 m}{\pi^{2}}\left(\frac{1}{p_{l_{0}}}+\frac{2 p_{l_{0}} a^{2}}{n^{2}}\right)
$$

After that the terms with $l= \pm l_{0}$ in the sum of Eq. (12) should be replaced by $\Pi_{n=2 l_{0}}$ from Eq. (13). Thus, for the $n$th harmonic of the screened potential at large $|z|$ we obtain the contribution from the point $k=0$ :

$$
\bar{V}_{n}(z)=\frac{\Gamma(n+1 / 2)}{\sqrt{\pi} n !} \frac{\tilde{e}^{2}}{z}\left(\frac{a}{z}\right)^{2 n}\left(1+\frac{\pi \kappa_{n}}{m a_{B} n}\right)^{-2} .
$$

The role of the effective dielectric constant for the $n$th harmonic is played by the quantity

$$
\varepsilon_{n}=\left(1+\frac{\pi \kappa_{n}}{m a_{B} n}\right)^{2}
$$

that tends to 1 with increasing $n$. The dielectric type of screening of harmonics with $n \geq 1$ can be understood in terms of the classical electrostatics (suggested by M.V. Entin). Each term of the series in Eq. (5) corresponds to the one of the expansion in multipoles: $n=1$ gives dipole-dipole contribution, $n=2$ - quadrupole-quadrupole and so on. At very large $|z|$ the fields of all multipoles on the nanotube surface are practically normal to the axis, hence, they cause only the displacements of electrons in the azimuthal direction $\varphi$. That is why the $z$-dependence of the $e-e$ interaction does not change; the system simply is polarized in accord with the dielectric mechanism when only the bound charges are available.

As to the oscillating part $\widetilde{V}_{n}(z)$ it is determined by zeros of the argument of logarithm in the Eq. (11). First of all one can see that such contributions exist not for all values of $n$. For a fixed concentration of electrons $p_{F} a \leq L$ whereas the singularity in the right-hand-side of Eq. (11) occurs only if $p_{F}^{2} a^{2}>(n-l)^{2}$ with $|l|<L$. Thus, the Friedel oscillations of the $n$th harmonic exist for $n \leq 2 L$ but for $n>2 L$ we have only nonoscillating contribution $\bar{V}_{n}(z)$ given by Eq. (14). The calculation of the oscillating part $\widetilde{V}_{n}(z)$ for $n \leq 2 L$ is totally similar to the derivation of Eq. (9). The singular points $k_{c}$ are given by the relation

$$
k_{c}=p_{l} \pm \sqrt{p_{F}^{2}-\left(\frac{n-l}{a}\right)^{2}}
$$

and the polarization operator at $k \rightarrow k_{c}$ takes the form

$$
\begin{aligned}
& \Pi \approx\left(\frac{m}{2 \pi^{2} k_{c}}\right) \ln \left|\frac{q_{l}}{k-k_{c}}\right|, \\
& q_{l}=\frac{2 k_{c} p_{l}\left(k_{c} p_{l} a^{2}+n l\right)}{n l\left(k_{c}-p_{l}\right)} .
\end{aligned}
$$

The result reads

$$
\widetilde{V}_{n}(z)=-\frac{2 \pi^{2}}{m z} \sum_{l} \frac{k_{c} \cos \left(k_{c} z\right)}{\ln ^{2}\left(z q_{l}\right)}
$$

Thus, only in the case $n \leq 2 L$ the Friedel oscillations in nanotubes give a noticeable contribution at large distances that is oscillating part of the $n$th harmonic of the 
screened potential decreases slower with increasing $z$ as compared with the regular part $\bar{V}_{n}(z)$. It is reasonable also that the Friedel oscillations look like superposition of the monochromatic waves with various periods $2 \pi / k_{c}$ (depending both on $n$ and $l$ ) rather than the single wave $\cos \left(2 p_{F} r\right)$ as it is in $2 \mathrm{D}$ and $3 \mathrm{D}$ systems.

The problem of the screened Coulomb potential in nanotubes was considered in [8]. However, the authors have given only numerical results for zeroth harmonic $\bar{V}_{0}(z)$ and did not discuss the Friedel oscillations.

To conclude, we have analyzed the $e-e$ interaction in nanotubes in the framework of the linear screening theory. Qualitative difference between quasi-1D systems and 3D and 2D systems is established. The Friedel oscillations in nanotubes do not determine the asymptotic behaviour of the screened potential at large distances in contrast with $3 \mathrm{D}$ and $2 \mathrm{D}$ systems.
This work has been supported by the RFBR, by the RF President grant for scientific schools, as well as by the Programs of the Russian Academy of Sciences.

1. T. Ando, A. Fowler, and F. Stern, Rev. Mod. Phys. 54, 437 (1982).

2. R. Egger and H. Grabert, Phys. Rev. Lett. 79, 3463 (1997).

3. Q. Yuan, H. Chen, Y. Zhang, and Y. Chen, Phys. Rev. B58, 1084 (1998).

4. R. Egger and H. Grabert, Phys. Rev. Lett. 75, 3505 (1995).

5. H.J. Schulz, Phys. Rev. Lett. 71, 1864 (1993).

6. M. Gell-Mann and K. Brueckner, Phys. Rev. 106, 364 (1957).

7. E.M. Lifshitz and L.P. Pitaevskii, Fizicheskaya Kinetika, Nauka, Moskva (1979), v. 10, § 40.

8. M.F. Lin and D.S. Chuu, Phys. Rev. B56, 4996 (1997). 